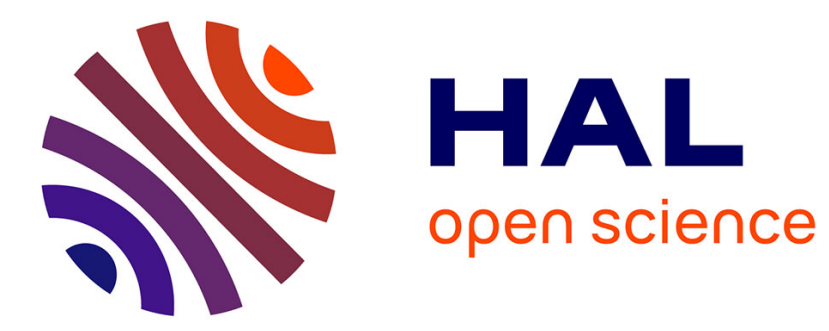

\title{
Transmission vibroacoustique par les doubles parois cylindriques
}

\author{
C. Cacciolati, M. Gotteland, M. Barbe, C. Lesueur
}

\section{To cite this version:}

C. Cacciolati, M. Gotteland, M. Barbe, C. Lesueur. Transmission vibroacoustique par les doubles parois cylindriques. Journal de Physique IV Proceedings, 1994, 04 (C5), pp.C5-139-C5-142. 10.1051/jp4:1994524 . jpa-00252927

\section{HAL Id: jpa-00252927 https://hal.science/jpa-00252927}

Submitted on 1 Jan 1994

HAL is a multi-disciplinary open access archive for the deposit and dissemination of scientific research documents, whether they are published or not. The documents may come from teaching and research institutions in France or abroad, or from public or private research centers.
L'archive ouverte pluridisciplinaire HAL, est destinée au dépôt et à la diffusion de documents scientifiques de niveau recherche, publiés ou non, émanant des établissements d'enseignement et de recherche français ou étrangers, des laboratoires publics ou privés. 


\title{
Transmission vibroacoustique par les doubles parois cylindriques
}

\author{
C. CACCIOLATI, M. GOTTELAND, M. BARBE et C. LESUEUR
}

Laboratoire Vibrations Acoustique, Bâtiment 303, Institut National des Sciences Appliquées, 20 Avenue Albert Einstein, 69621 Villeurbanne cedex, France

Résumé : A theoretical model is established to study the direct transmission of sound through a finite cylindrical double shell. Shells comply with DONNELLS's equations and the theory include constantly spaced stiffeners in axial and circumferential directions. However this is limited in the case of large number of stiffeners. The acoustical excitation is a plane wave located in an infinite medium outside the shell. The two internal media are homogeneous. Absorption is taken into consideration by a complex wave number. The fluid structure coupling is exactly modelised and mechanical links between shells are constant distributed stiffness along circumferential lines. The solution of the equations is obtained by a modal method. Numerical results show the comparison between structural linkage and air gap effetcs on the transmission of sound inside the inner cavity.

\section{INTRODUCTION}

Il s'agit de modéliser la transmisson du son de l'extérieur vers l'intérieur, à travers les parois cylindriques doubles. La bibliographie sur ce sujet est analysée dans les publications antérieures : CACCIOLATI (1989) et CACCIOLATI - GOULAIN (1990). Nous présentons ici l'évolution du modèle qui prend en compte l'absorption acoustique du milieu intercoque et la transmission par voie solide.

\section{MODELE}

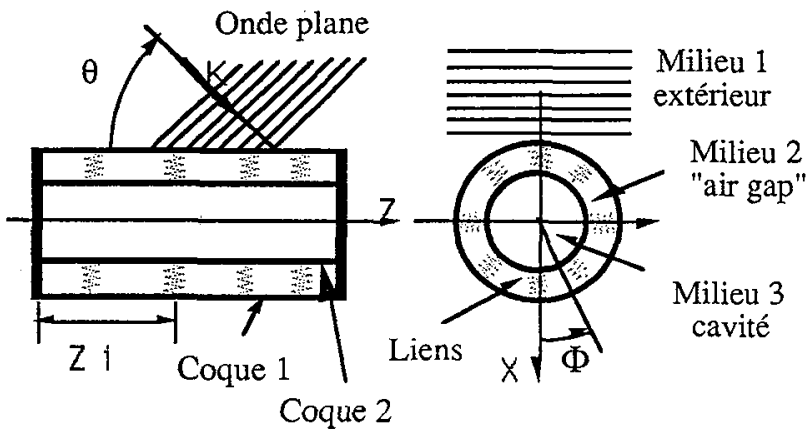

Figure $\mathrm{N}^{\circ} 1$
L'excitation est une onde plane harmonique qui se propage dans le milieu extérieur avec une incidence $\theta$ par rapport à la génératrice des cylindres(figure1). Nous considérons deux coques de longueur $\mathrm{L}$, de rayon voisin a1 et a2, simplement appuyées à leurs extrémités. L'impédance $\mathrm{Z}^{\text {sh }}$ des coques est supposée connue.

Les milieux intérieurs sont fermés par des fonds rigides. L'absorption dans le milieu intercoque est prise en 
compte par le nombre d'onde complexe de DELANY et BAZLEY. Dans la cavité on considère un facteur de perte expérimental. Des liens élastiques radiaux de raideur linéïque cte $\mathrm{K}$ sont uniformément répartis sur des circonférences aux cotes $\mathrm{z}_{\mathbf{i}}$ aléatoires. L'équilibre du système se traduit par :

$$
\begin{aligned}
& \mathrm{Z}^{\text {sh1 } \dot{\mathrm{W}} 1}=\mathrm{P}^{\text {gap }}(\mathrm{a} 1)-\mathrm{P}^{\text {ext }}(\mathrm{a} 1) \\
& \mathrm{Z}^{\text {sh2 }} \dot{\mathrm{W} 2}=\mathrm{P}^{\text {cav }}(\mathrm{a} 2)-\mathrm{P}^{\text {gap }}(\mathrm{a} 2)
\end{aligned}
$$

Les pressions acoustiques sont notées $\mathrm{P}$. Les indices supérieurs se rapportent aux éléments du système: sh coques, 1 ou "ext" milieu externe, 2 ou "gap" intercoque, 3 ou "cav" intérieur. Les déplacements des coques sont notés: W1 pour la coque 1 (extérieure), W2 pour la coque 2 (intérieure). Les rayons moyens des coques sont a1 et a2. L'équilibre de chaque coque traduit le principe fondamental de la mécanique suivant la direction radiale. La normale aux surfaces est orientée positivement vers l'extérieur. La pression acoustique sur les surfaces des coques est définie en coordonnées cylindriques, elle satisfait l'équation d'Helmohltz. Ces fonctions sont explicitées dans la bibliographie classique ou dans les publications citées en référence.

\section{EQUATIONS D'EQUILIBRE ET SOLUTION}

Les équations d'équilibre modales du double cylindre s'obtiennent en développant les pressions internes de (1) en série sur les bases modales de cavité et les autres termes sur la base modale des coques. On projette ensuite les impédances modales acoustiques dans la base modale des coques qui est prise comme base de calcul. Nous notons $(\wedge)$ les quantités exprimées dans la base acoustique et $\mathrm{D}$ la matrice de projection de la base acoustique sur la base des coques. Pour chaque ordre circonférentiel $\mathrm{m}$ il faut résoudre le système suivant. Pour simplifier l'écriture, l'indice $\mathrm{m}$ de l'ordre circonférentiel est sous entendu, l'ordre axial du mode de coque est $n=1,2, \ldots \mathrm{N}$, celui de l'ordre axial des modes acoustiques est $\mathrm{q}=0,1, \ldots \mathrm{N}-1$. Dans le système suivant, excepté $\mathrm{ZK}$ toutes les autres matrices d'impédance sont diagonales car exprimées dans leur base propre. Le couplage intermodal est dû à $D$.

$$
\left[\begin{array}{cc}
{\left[D \hat{z}_{q}^{11} D^{-1}-z_{n}^{\text {sh1 } \left.1-Z K_{n}^{q}-z_{n}^{\text {ray }}\right]}\right.} & {\left[D \hat{z}_{q}^{12} D^{-1}+Z_{K}^{q}\right]} \\
{\left[-D \hat{z}_{q}^{21} D^{-1}+Z K_{n}^{q}\right]} & {\left[D\left(\hat{z}_{q}^{\text {int }}-\hat{z}_{q}^{22}\right) D^{-1}-z_{n}^{\text {sh2 } 2}-Z K_{n}^{q}\right]}
\end{array}\right]\left[\begin{array}{c}
\dot{W} 1_{n} \\
\dot{W} 2_{n}
\end{array}\right]=\left[\begin{array}{c}
P b l o q_{n} \\
\\
0
\end{array}\right]
$$

Chacun des quatres blocs de matrices est une matrice de taille $\mathrm{N} \times \mathrm{N}$ où "N" est l'ordre maximum du développement axial sur la base propre des coques. On remarque que ces matrices bloc sont des matrices pleines. Une fois la solution W1 $\mathrm{n}$ et $\mathrm{W} 2 \mathrm{n}$ connue, les amplitudes vibratoires sont ensuite obtenues par sommation sur les ordres circonférentiels. Les pressions pariétales en tout point du système sont alors connues.

Le point particulier de cette formulation est le calcul de $\mathrm{ZKqn}$. et $\mathrm{Z}^{\mathrm{ij}} \mathrm{n}$ chapeau Dans le milieu intercoque, les coques reçoivent une pression qui est la somme de la pression acoustique due à la lame d'air et de la pression mécanique due aux liens et au matériau absorbant. Dans la pratique ce milieu contient de la laine de verre, l'absorption qui en découle est représentée par un nombre d'onde complexe. La pression acoustique est exprimée dans la base acoustique. Le principe du calcul des impédances modales acoustiques $\mathrm{Z}^{\mathrm{ij}}$ n chapeau est donné dans [1] et leurs expressions sont données ci-dessous (3) dans les cas de ce modèle dit "exact" à ceci près qu'il n'y a pas de modèle connu pour le nombre d'onde à utiliser. Le modèle de DELANY-BAZLEY [3] qui est valable pour une onde plane polarisée suivant la direction normale est utilisé en pis-aller. La distorsion du modèle d'absorption par les ondes cylindriques est alors inévitable. Le 
quadripôle d'impédances acoustiques qui relie (Pa1, Pa2) aux vitesse des coques (W1, W2) dans le cas des ondes cylindriques est donné par (3). Nous comparerons cette formulation avec les impédances acoustiques

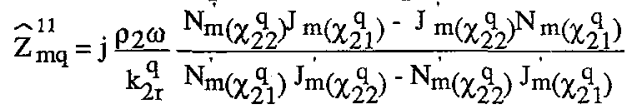

$$
\hat{z}_{m q}^{12}=-j \frac{2 \rho_{2} \omega}{\pi \chi_{21}^{q} k_{2 r}^{q}} \frac{1}{N_{m}^{\prime}\left(\chi_{21}^{q}\right)^{\top} \cdot\left(\chi_{22}^{q}\right)^{-N_{m}^{\prime}\left(\chi_{22}^{q}\right)^{\prime}{ }_{m}^{\prime}\left(\chi_{21}^{q}\right)}}
$$

$$
\hat{Z}_{m q}^{21}=-j \frac{2 \rho_{2} \omega}{\pi \chi_{22}^{q} k_{2 r}^{q}} \frac{1}{N_{m}^{\prime}\left(\chi_{21}^{q}\right)^{\prime}} \frac{1}{m}\left(\chi_{22}^{q}\right)^{-N_{m}^{\prime}\left(\chi_{22}^{q}\right)^{J_{m}^{\prime}\left(\chi_{21}^{q}\right)}}
$$

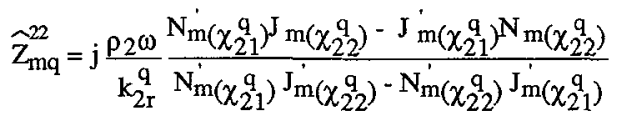

d'une onde plane normale (4) sur une couche de laine de verre d'épaisseur égale à la distance intercoque. Dans ce cas, l'impédance acoustique pour tout ordre circonférentiel et tout ordre radial est proche de celle des ondes acoustiques d'incidence normale. Au cours de l'exposé nous comparerons les deux approches analytiques avec les expériences en onde plane et en onde cylindriques:

$\widehat{Z}_{\mathrm{mq}}^{1 \mathrm{I}}=\widehat{\mathrm{Z}}_{\mathrm{mq}}^{22}=\left(\mathrm{Zr}_{\infty}+\mathrm{j} \mathrm{Z} \mathrm{i}_{\infty}\right) \operatorname{coth}[(\alpha+\mathrm{j} \beta) \mathrm{d}]$

$$
\widehat{Z}_{m q}^{12}=\widehat{Z}_{m q}^{21}=\frac{\left(Z_{r_{\infty}}+j Z_{\infty}\right)}{\sinh [(\alpha+j \beta) d]}
$$

La pression mécanique dues aux liens est une projection classique des forces linéiques dues aux $\mathrm{N}$ liens sur la base modale des coques, elle donne lieu à l'impédance $\mathrm{ZKG}_{\mathrm{mn}}(5)$. L'effet mécanique de la structure de l'absorbant (à réaction localisée) peut être pris en compte par un modèle de poutre en vibrations axiales.

$$
\mathrm{ZK}_{\mathrm{mn}}^{\mathrm{q}}=\sum_{\mathrm{i}=1}^{\mathrm{N}} \frac{\mathrm{K}_{\mathrm{i}}^{*}}{\mathrm{jL} \omega \varepsilon \mathrm{n}} \sin \left(\frac{\mathrm{n} \pi \mathrm{z}_{\mathrm{i}}}{\mathrm{L}}\right) \sin \left(\frac{\mathrm{q} \pi \mathrm{z}_{\mathrm{i}}}{\mathrm{L}}\right)
$$

\section{RESULTATS}

Dans le cas d'un fuselage d'avion soumis à une excitation aérienne le résultat intéressant est l'isolement brut $\mathrm{Db}$ (ou NR: noise reduction). Il exprime en décibels le rapport entre les valeurs quadratiques moyennes de la pression sur la surface externe et de la pression dans le volume. On effectue le calcul des valeurs quadratiques moyennées sur le temps et sur l'espace. (* indique le conjugué du nombre complexe).

$$
\left\langle\overline{\mathrm{P}}^{\mathrm{ext}}{ }^{2}\right\rangle=\sum_{\mathrm{mn}} \varepsilon_{\mathrm{m}} \varepsilon_{\mathrm{n}}\left\|\mathrm{P}_{\mathrm{mn}}^{\mathrm{ext}}\right\|^{2} \text { et }\left\langle\overline{\mathrm{P}^{\mathrm{cav}}}{ }^{2}\right\rangle=\sum_{\mathrm{mq}} \varepsilon_{\mathrm{m}} \varepsilon_{\mathrm{q}} \mathrm{L}\{\mathrm{m}, \mathrm{a} 2\}\left\|\mathrm{D}_{\mathrm{mq}}\right\|^{2}
$$

Les prévisions sont effectuées sur une maquette cylindrique à double parois : a1=0.4705 m, a2=0.401 m. La coque extérieure d'épaisseur $0.5 \mathrm{~mm}$, en $\mathrm{AU} 4 \mathrm{G}$ est raidie circonférentiellement par 11 cadres équidistants. La coque intérieure est un tricouche de $6 \mathrm{~mm}$ d'épaisseur représenté par un modèle homogénéisé $\left(E=4.0410^{9} \mathrm{~Pa}, \rho=517 \mathrm{Kg} / \mathrm{m} 3\right)$. La longueur de l'ensemble est de $1.78 \mathrm{~m}$.

Si le milieu intercoque ne contient pas d'absorbant, l'isolement acoustique est faible et peu dépendant de la raideur des liens, voir figure 2a. Dans ce cas l'impédance de transfert de la lame d'air est grande par rapport à celle de la coque intérieure et celle-ci "suit" la coque extérieure. Elle a simplement un effet de masse rajoutée. La courbe de plus faible isolement pour $\sigma=0$ Rayls/m et 11 liens de 13 e+5 $\mathrm{N} / \mathrm{m}^{2}$.est très peu affectée par une modification de $\mathrm{K}$.

Si le milieu intercoque est absorbant, l'isolement acoustique augmente. La figure $2 \mathrm{~b}$ montre l'influence très significative des liens intercoque sur l'isolement de la double paroi cylindrique. Entre 0 et $\mathrm{f} 1$ $\mathrm{Hz}$ ils réduisent d'autant plus l'amélioration donnée par le matériau absorbant qu'ils sont raides. Avant $\mathrm{f} 1$ l'impédance des liens est supérieure à l'impédance acoustique spécifique $\rho_{2}$ c2 du milieu intercoque, la transmission solidienne domine (sur la figure $2 b, f]=1120 \mathrm{~Hz}$ avec 11 liens de $K=510^{5} \mathrm{~N} / \mathrm{m}^{2}$ ).

$$
\mathrm{f}_{1}=\mathrm{KNK} / 2 \Pi \mathrm{L} \mathrm{\rho}_{2} \mathrm{c}_{2}
$$




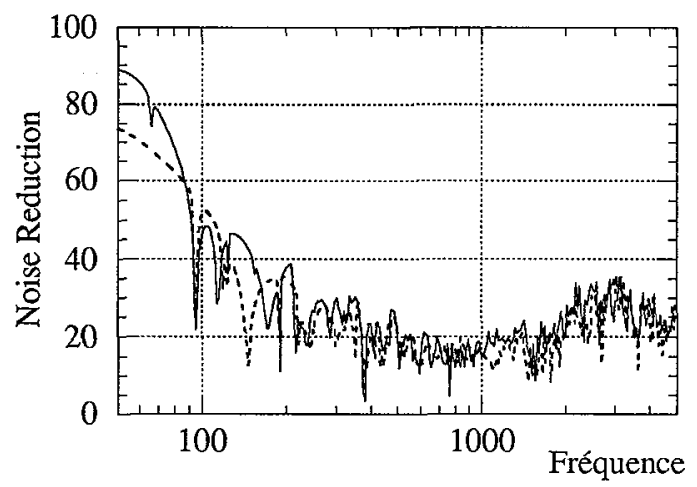

CALCUL maquette double coque onde cylindrique intercoque incidence 45 degrès, sans absorbant - sans liens radiaux

11 liens radiaux $\mathrm{K}=1310^{5} \mathrm{~N} / \mathrm{m} / \mathrm{m}$

Figure $\mathrm{N}^{\circ} 2$ a

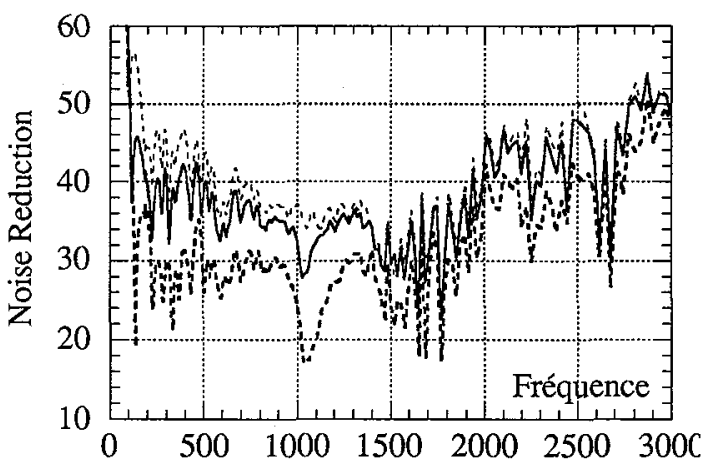

CALCUL maquette double coque 11 liens circonférentiels intercoque incidence 45 degrès, absorbant 9000 Rayls $/ \mathrm{m}$

$$
\begin{aligned}
& K=0 \mathrm{~N} / \mathrm{m}^{2} \\
& K=10^{5} \mathrm{~N} / \mathrm{m}^{2} \\
& \mathrm{~K}=510^{5} \mathrm{~N} / \mathrm{m}^{2}
\end{aligned}
$$

Figure $\mathrm{N}^{\circ} 2 \mathrm{~b}$

Remerciements:Ce travail a été financé par le s ${ }^{\mathrm{ce}}$ Acoustique de l'AEROSPATIALE - Toulouse - Avions

NOTATIONS :

$\mathrm{C}_{2}$ célérité du son dans le fluide

$\sigma \quad$ résist. spécifiq du matér absorbant (Rayls/m)

$\rho_{2}$ masse volumique du fluide

$\chi_{21}$ argument $=\mathrm{k}_{2 \mathrm{r}}$ a1, $\quad \chi_{22}$ argument $=\mathrm{k}_{2 \mathrm{r}}$ a2

$\mathrm{n}$ ordre axial des modes de cavité, de coque

$\mathrm{Z}_{\mathrm{r} \infty}=\rho_{2} \mathrm{C}_{2}\left[1+0.057\left[\frac{\sigma}{\rho_{2} \mathrm{f}}\right]^{0.75}\right]$

$$
\varepsilon_{\mathrm{m}}=1 \quad(\operatorname{resp}=0.5) \text { si } \mathrm{m}=\mathrm{O}(\operatorname{resp} \text { si } \mathrm{m} \neq 0)
$$

$$
\mid \begin{array}{ll}
\wedge & \text { quantités mesurées dans la base de la cavité } \\
\hat{\mathfrak{w}} & \text { vitesse particulaire radiale du fluide dans la } \\
& \text { base des modes de la cavité }
\end{array}
$$

W la vitesse radiale de la coque dans la base des modes de la coque

$Z_{\mathrm{i} \infty}=\rho_{2} C_{2}\left[0.087\left[\frac{\sigma}{\rho_{2} f}\right]^{0.73}\right]$

$$
k_{2}=\frac{\omega}{C_{2}}\left[\left(1+0.098\left[\frac{\sigma}{\rho_{2} f}\right]^{0.70}\right)-j\left(0.19\left[\frac{\sigma}{\rho_{2} f}\right]^{0.59}\right)\right]
$$

\section{BIBLIOGRAPHIE}

[1] CACCIOLATI C. Sound transmission through a double walled cylindrical shell with radial elastic links, ASA, 19th meeting, May 1990, Pennstate, U.S.A.

[2] CACCIOLATI C., GOULAIN M. Study model for the reduction of noise through double wall cylindrical shell. Comparison between theory and experiment in light fluid. Congrès INTERNOISE, Août 1990, Gotteborg, Suède.

[3] DELANY M.E., BAZLEY E.N. "Acoustical properties of fibrous absorbent materials." Applied Acoustics, vol. $\mathrm{n}^{\circ} 3,1970$. 